\title{
Rezipienten ohne Bewußtsein?
}

\author{
von Michael Schmolke
}

Kurz nach der deutschen Bundestagswahl 1976, als die Grundgedanken dieses Beitrags für einen Vortrag bei der Jahrestagung der Gesellschaft Katholischer Publizisten Deutschlands ${ }^{1}$ entstanden, lag es nahe, die Fragestellung des ursprünglich vorgeschlagenen Themas („Der Leser - Opfer oder Partner der Medien?“) zu verändern: Der Staatsbürger, Opfer oder Partner der Politik? Wer sich an die spannenden Stunden der Hochrechnungen erinnert, wird sich des Eindrucks nicht erwehren können, daß der Staatsbürger, von Kritikern des parlamentarischen Systems gern zum Stimmvieh abgewertet, keineswegs Opfer ist: Als Wähler zeigt er, selten zwar, aber vielleicht oft genug, daß er doch über so viel Macht verfügt, wie sie zur Ausübung der Partnerrolle notwendig ist.

Damit aber kommen wir zum Unterscheiden: Dem Staatsbürger ist diese Macht verfassungsmäßig und von Gesetzes wegen verbrieft; dem „Leser", wenn er mit irgendetwas Macht-Ahnnlichem ausgestattet sein sollte, was ihn Partner zu sein befugt, ist beinahe nichts verbrieft.

Meine These sei nun ohne lange Vorrede an den Anfang gestellt: Auch ohne konkrete Verbriefung hat der Leser, richtiger: haben die Leser, so etwas wie Macht, und insofern können sie Partner der Medien sein. (Das schließt nicht aus, daß sie gelegentlich auch ihre Opfer sind; paradoxerweise - mehr davon unten - ergibt sich aus einigen Bestandteilen dieser "Opfer"-Rolle wiederum „Macht".)

Bevor wir der Frage im einzelnen nachgehen, noch eine notwendige Klarstellung: Den "Leser" als solchen gibt es ja heute nicht mehr bzw. nur noch ganz selten. Die Gesellschaft Katholischer Publizisten hat bei ihrer Formulierung des Konferenzthemas einen Kompromiß geschlossen:Neben dem "Leser", dem klassischen Rezipienten-Typ der "bürgerlichen Ơffentlichkeit", stehen „die Medien“ im Plural: Ihnen, den "großen Vier", ist der Leser ausgesetzt, und wenn er wirklich liest, so ist dies nur eine Ausdifferenzierung seiner Rolle als Teilnehmer am „Zeitgespräch der Gesellschaft", die Rolle, die wir als ganze bezeichnen könnten mit Begriffen wie Mediennutzer oder, wenn das zu positiv klingt, Medienkonsument.

Aber selbst der lesende Leser - gemeint ist der Presse-Leser - der Gegenwart "liest" nicht nur: Er rezipiert eigentlich ständig „Kon-Texte“ im wörtlichen Sinne: Nachdem er sich der im Auto-Radio gebörten Meldung vom samstäglichen Sieg seines Fußball-Vereins in der Tagesschau vergewissert hat, vergnügt er sich spätestens im Aktuellen Sportstudio optisch am geliehenen Erfolgserlebnis, was ihn nicht daran hindert, am Sonntag oder spätestens Montag in der Zeitung nachzulesen, wovon er gehört und was er - mediatisiert - gesehen hat: die dokumentarische Qualität der Presse, über die progressive Historiker am Anfang unseres Jahrhunderts noch diskutieren mußten ${ }^{2}$, hat erst durch die schnelleren elektronischen Medien ihren vollen Stellenwert geoffenbart.

Wenn ich also im weiteren der Kürze halber gelegentlich vom Leser spreche, so meine ich damit in der Regel den multiplen Medienangebots-Nutzer. 
Zurück zu meiner These, der Leser habe Macht, was nicht ausschließe, daß er Opfer werden könne. Für diese These möchte ich mit Hilfe folgender fünf Ansätze Argumente sammeln:

1. Die geschriebenen Rechte des Lesers (Rezipienten).

2. Die ökonomische Machtposition des Lesers.

3. Wir wissen viel über den Leser, und das macht ihn stark.

4. Der Drang, noch mehr über den Leser (Rezipienten) zu erfahren, hält an.

5. Schließlich eine Frage: Hat der Leser Pflichten, durch deren Erfüllung er eine Position mindestens der moralischen Stärke aufbauen könnte?

\section{Geschriebene Rechte des Rezipienten}

Von Rechten zu reden, geschieht im Zusammenhang mit unserem Thema insofern zu recht, als Rechte Macht verleihen. Das ältere klassische Presserecht wie auch neuere Entwürfe verbriefen Rechte des Lesers vorzugsweise dort, wo vom Schutz der Persönlichkeit, der Ehre, der Intimsphäre, die Rede ist. Im möglicherweise modernsten Mediengesetzentwurf unseres Kulturkreises, der österreichischen „Regierungsvorlage zum Mediengesetz ${ }^{\text {"3 }}$, ist ein sehr umfangreicher $\$ 14$ der "Verletzung des höchstpersönlichen Lebensbereiches" gewidmet. Aber bei unserer Fragestellung geht es uns ja nicht in erster Linie um die rechtlich zu sichernde Schutzwürdigkeit der Person als Person, sondern um die spezifischen Rechte des Lesers in seiner Rollenausübung als Leser, als Rezipient. Und im Hinblick darauf ist nur ein Recht national und international kodifiziert worden: das sogenannte „Recht auf Information“.

Wir finden es klassisch formuliert, wenngleich weitgehend wirkungslos geblieben, in der Allgemeinen Erklärung der Menschenrechte der UNO von 1948 im Artikel 19: "Jeder Mensch hat das Recht auf freie Meinungsäußerung; dieses Recht umfaßt die Freiheit, Meinungen unangefochten anzuhängen und Informationen und Ideen mit allen Verständigungsmitteln ohne Rücksicht auf Grenzen zu suchen, zu empfangen und zu verbreiten."

Wir finden das Recht auf Information, vorsichtiger formuliert, im Art. 10,1 in der "[Europäischen] Konvention zum Schutze der Menschenrechte und Grundfreiheiten“ von 1950 (in der Bundesrepublik Gesetz seit 1952, in Osterreich Bestandteil der Verfassung). Wir finden es als Recht, „sich aus allgemein zugänglichen Quellen ungehindert zu unterrichten “, in Art. 5,1 des Grundgesetzes, und als „Freiheit, unbehindert von behördlichen Eingriffen und ohne Rücksicht auf Bundes- und Landesgrenzen Informationen und Ideen aller Art in Wort, Schrift, Druck, Bild, Ton oder in einem anderen Verständigungsmittel eigener Wahl zu suchen, zu empfangen ...“, im Art. I, $\mathbb{1}$ (1) des österreichischen Mediengesetzentwurfs (RV).

Das Recht auf Information ist jedoch noch immer ein relativ wenig konkretes Recht, wie es z. B. aus der Einschränkung "allgemein zugängliche Quellen“ ausgerechnet im bundesdeutschen Grundgesetz erkennbar wird. Es ist ein Recht, das jedermann zukommt und das dennoch oft sogar von den Spezialisten und Professionellen auf dem Gebiet der Informationsbeschaffung, den Journalisten, nur mit Mühe realisiert werden kann. Insbesondere die Administration der Staaten und Gemeinden, aber auch gesell- 
schaftlicher Großorganisationen wie z. B. der Kirche, setzen der Inanspruchnahme dieses Rechtes hinhaltenden Widerstand entgegen. Die Inflation allenthalben neu entstandener Pressestellen wird ja von nicht wenigen Journalisten als Installation elastischer Informationsbarrieren interpretiert.

Das Recht auf Information ist insbesondere, wie zuletzt das zähe Feilschen auf der Konferenz für Sicherheit und Zusammenarbeit in Europa (KSZE) gezeigt hat, im internationalen, ideologisch beschwerten Rahmen nur mühsam zu konkretisieren. Die KSZE-Schlußakte bekennt sich zwar zur Anerkennung der Menschenrechte und Grundfreiheiten, aber dort, wo es konkret um Verbesserung der Informationschancen für alle Europäer geht, suchen wir vergeblich den Satz, daß jeder Bürger eines jeden Staates das Recht habe und die Möglichkeit bekommen solle, jede gewünschte Zeitung eines jeden anderen europäischen Landes zu beziehen und zu lesen; stattdessen ist von bescheidenen Verbesserungen der Arbeitsbedingungen für Journalisten die Rede.

Der kanadische Kommunikationswissenschaftler John W. Mole hat auf die Schwierigkeiten der allgemeinen Anerkennung eines internationalen Rechtes auf Information hingewiesen ${ }^{4}$ und hat - meines Erachtens zutreffend - geurteilt, daß bislang nur eine einzige internationale Großorganisation, die für alle ihre Mitglieder Recht setzen kann, nämlich die Römisch-katholische Kirche, das Recht auf Information in einem quasi legislativen Dokument, nämlich dem vielgeschmähten Konzilsdekret „Inter mirifica" (1963) fast wie ein Naturrecht anerkannt hat: "Inest ergo in societate humana ius ad informationem ..." heißt es lapidar im Art. 5 des Dekrets. Dies gilt, jedenfalls der Intention nach, für alle Menschen, kirchenrechtlich aber doch bestimmt für alle Katholiken, die diesen Anspruch mindestens gegenüber den Institutionen der eigenen Kirche mit Aussicht auf Erfolg zu verwirklichen versuchen sollten.

Die Pastoralinstruktion "Communio et progressio" 5 hat das Recht auf Information in den Nummern 33-47 in bemerkenswerter Weise entfaltet, in manchen Punkten so konkret, daß man jeden, der etwas Herzhaftes über dieses Recht erfahren will, mit gutem Gewissen auf "Communio et progressio" verweisen kann. (Über die den Rechten entsprechenden Pflichten, die in "Inter mirifica " und "Communio et progressio" notiert werden, wird im 5. Abschnitt zu reden sein.) Mit großem Nachdruck haben übrigens sowohl die Päpstliche Kommission für die Instrumente der sozialen Kommunikation als auch der in publizistischen Dingen sehr aufmerksame Papst Paul VI. mehrfach auf das Recht auf Information hingewiesen, so zuletzt in den Stellungnahmen zum 10. Welttag der sozialen Kommunikationsmittel 1976, wo Paul VI. davon spricht, daß es unter den grundlegenden Rechten des Menschen eines gebe, „das fast ausschließlich von den sozialen Kommunikationsmitteln selbst abhängt: das Recht auf richtige und vollständige Information ${ }^{* \theta}$.

(Diese römisch-weltkirchlichen Bezüge werden hier nicht deshalb betont, weil das ursprüngliche Auditorium die Gesellschaft Katholischer Publizisten war, sondern weil die Kirche auf diesem einen Gebiet fortschrittliche Markierungen gesetzt hat, die von der gesellschaftlichen und politischen Wirklichkeit der Staaten und der Kirche selbst erst noch eingeholt werden müssen.)

\section{Die ökonomische Machtposition des Rezipienten}

Es ist bekannt, daß die meisten privatwirtschaftlich produzierten Medien ihre Erlöse zum guten Teil nicht aus dem Verkauf ihrer Produkte an den Endverbraucher erzielen, 
sondern aus dem Verkauf von Raum (Druckfläche) oder Zeit zu Werbezwecken. Der Wert und damit der Preis z. B. des Anzeigenraums orientiert sich an verschiedenen Daten, deren grundlegende auch heute noch die Zahl und die Qualität der hochwahrscheinlich erreichbaren (im Falle der hier als Beispiel gewählten Presse:) Leser ist. Der Leser also ist dem Verleger teuer, weil er den Anzeigenraum seines Blattes wertvoll machen hilft. Pressejournalisten wissen um dieses Abhängigkeitsverhältnis, und wo sie es verleugnen, werden sie vom Kaufmann im Hause mehr oder minder energisch darauf hingewiesen.

Gegenüber Presseprodukten hat nämlich der Rezipient in unserer Kommunikationsverfassung ein Recht, das ich, vergleichbar dem Budgetrecht der frühen Parlamente, sein vornehmstes nennen möchte: das Recht, eine Zeitung oder Zeitschrift zu kaufen oder nicht zu kaufen. Gegenüber den Angeboten der öffentlich-rechtlichen Rundfunkanstalten hat er dieses Recht nicht: Gebühren zahlen muß er in jedem Falle. Seine Freiheit besteht im An- oder Abschalten, nicht aber in der materiell fühlbaren Verweigerung.

Die Verweigerung durch Abschalten wird freilich auch für den Rundfunk indirekt überall dort fühlbar, wo er trotz öffentlich-rechtlicher Verfaßtheit das illegitime Kind kapitalistisch zu betreibender Hörfunk- bzw. Fernsehwerbung in die Welt gesetzt hat. Viele und qualitativ gute Hörer/Seher im unmittelbaren Umfeld der Werbezeiten machen diese Zeiten zu hohen Preisen verkäuflich. Ein gewichtiger Anstoß der vom Rundfunk bei uns zulande mit relativ viel Aufwand betriebenen Hörer- und Seherforschung kommt aus den $Z$ wängen der Werbung. Erst recht gilt dies natürlich für die Leserforschung der Druck-Medien: Kein Werbetreibender kauft noch - und schon lange nicht mehr - eine Katze im Sack, sondern nur noch eine schöne und große Katze, die ihm verrät, wie schnell und wie oft sie wie viele und wie wohlbepelzte Mäuse fangen kann.

\section{Wir wissen viel über den Leser, und das müßte ihn eigentlich stark machen}

Wichtige Anstöße zur Leser- und im weiteren Sinne Publikumsforschung kamen einerseits aus der Wählerforschung ${ }^{7}$ und andererseits aus dem Wissensdrang der Werbewirtschaft. Dem erstgenannten Ansatz verdankt die Wirkungsforschung ihre wichtigsten Anstöße, aus dem zweiten entstand all das, was wir heute "Media-Forschung “ nennen. Die Fragestellungen lauteten einmal - ich vergröbere stark: Wieviele Menschen ändern unter welchen Einflüssen ihre Meinung? Und im zweiten Falle: Wer liest, hört, sieht wann, was, wie lange und mit welchem Kostenaufwand, und wie sieht es in seinem Kopf, seinem Geldbeutel, seiner Wohnung und seiner Garage aus? (Mit der Hintergrundfrage: Wem kann man sinnvollerweise welche Bedürfnisse zu haben vorschlagen?)

Aus der Mediaforschung im rein werblichen Verständnis wurde, nicht zuletzt durch die Rundfunkanstalten, eine Publikumsforschung im weiteren Sinne entwickelt, die uns heute hoch-differenziertes Fakten-, Daten- und auch Motivwissen in erstaunlicher Fülle anbietet.

Frei vom Impetus Werbung, stattdessen aus pastoral-publizistischen Erwägungen, hat die Arbeitsgemeinschaft Katholische Presse 1974/75 mit ihrer "Feldbefragung " ${ }^{8}$ einen anderen Typ von Leserforschung erprobt, bei dem die Frage nach den Erwartungen und Bedürfnissen der katholischen Bevölkerung gegenüber einem bestimmten Presse- 
typ, nämlich den diözesanen Kirchenzeitungen, im Mittelpunkt stand. Auch diese Untersuchung war nicht ganz uneigennützig, galt es doch, dem allmählichen Auflagenschwund durch Eingehen auf zuvor ungekannte Erwartungen der Rezipienten nach Kräften entgegenzuwirken. Schließlich haben zahlreiche rein wissenschaftlich motivierte Einzeluntersuchungen das große Feld Publikum mindestens in einigen Eckchen erhellt.

Sehr kurz gefaßtes Fazit, das die Kenntnis der hier nicht referierbaren Ergebnisse voraussetzt: Wenn der Rezipient, wenn das hochverehrte Publikum wüßte, wieviel die Macher seiner liebsten Freizeitangebote über die Abnehmer wissen und immer noch zu wissen begehren, so würde sein Selbstbewußtsein ganz erheblich gefestigt werden. Wie kann einer „Opfer" sein, dem soviel Aufwand gilt? Und selbst wenn ein großer Teil dieses Aufwands nur getrieben wird, um den Leser als Käufer oder Wähler, also doch als "Opfer" zu erreichen, zeigt die von Politik und Wirtschaft anerkannte Notwendigkeit dieses Aufwands, daß der Rezipient im Grunde als Macht eingeschätzt wird. Nur: In der Regel weiß er es nicht. Und Nicht-Opfer sein, bedeutet noch nicht Partner sein.

\section{Der Drang, noch mebr über den Rezipienten zu erfabren bält an}

Mit dieser Feststellung ist nicht nur die Tatsache gemeint, daß die werbetreibende Wirtschaft und die für sie arbeitenden Werbeagenturen es ganz selbstverständlich gewohnt sind, Jahr für Jahr generelle und spezielle Media-Analysen präsentiert zu bekommen. Fragestellung und Methodenapparat werden immer noch verfeinert. Bald wird man vom Leser wirklich haarklein wissen, wie er sich räuspert und wie er spuckt, - denn selbst aus diesen bislang beiläufig erscheinenden Tätigkeiten lassen sich möglicherweise Anhaltspunkte für die Konzeption einer neuen Zielgruppenzeitschrift gewinnen. Neuerdings wenden sich wieder große Verlage diesem besonderen publizistischen Spekulationsfeld der auf eine verhältnismäßig kleine, aber wirtschaftlich leistungsfähige Zielgruppe gerichteten Zeitschrift zu. Die Spekulation wird jedoch keineswegs spekulativ betrieben, sondern nach sorgfältiger Erforschung des "Opfers", das doch auf der anderen Seite schon 1951 von Johann Wilhelm Naumann ahnungsvoll als „Majestär Leser" bezeichnet wurde".

Aber auch die nicht (oder nicht primär) wirtschaftlichen Zwecken dienende Rezipienten-Forschung steht nicht still. Wenn heute große Rundfunkanstalten ihre Programmstruktur zu ändern überlegen, gehen diesen Entscheidungen, wie es z. B. 1973 beim ZDF der Fall war, sorgfältige Untersuchungen der Tagesablauf- und Rezeptionsgewohnheiten des Publikums voran ${ }^{10}$. Auch die routinisierte Fernsehteilnehmerforschung, wie sie früher mit dem fast zum Schlagwort gewordenen Namen "Infratest" und seit 1975 mit dem neuen Verfahren "Teleskopie“ kurz bezeichnet wird, ist in Entwicklung begriffen. Die mittels Teleskopie gewonnenen Erkenntnisse über „Kinder vor dem Bildschirm "11 haben in den Sommermonaten des Jahres 1976 großes öffentliches Aufsehen erregt. Zwar bestätigten sie nur längst Vermutetes, aber das Bestätigte wird sich nun nicht mehr so leicht von der Hand weisen, das Argument "Kind vor dem Bildschirm" sich nicht als ein kapitalistisch motiviertes entwerten lassen.

Auch die seit 1974 in der gemeinsamen Trägerschaft von ARD und ZDF und Bundeszentrale für politische Bildung durchgeführte Untersuchung „Fernsehen als Sozialisationsfaktor", bezogen auf die Situation in Familien, läßt mit gutem Grund vermuten, 
daß der Rezipient nicht als ökonomischer Machtfaktor (und insofern möglichst als "Opfer"), sondern als Partner gesehen wird".

Auf der anderen Seite haben die jüngsten Studien und Anregungen von Elisabeth Noelle-Neumann, die sich dem Stichwort „Schweigespirale“ zuordnen lassen, deutlich gemacht, daß sich die Position des "Lesers", seitdem er auch Fernsehzuschauer ist, durch dieses neue Medium entscheidend verändert hat, und $\mathrm{zwar} z u$ seinen Ungunsten ${ }^{13}$. Jenes Medium, das man im Unterschied zur Zeitung nicht umblättern kann, mindert im Verein mit dem Konsonanz-Streben vieler Journalisten die Selektionschancen des Publikums: Damit verstärken sich Vermutungen in Richtung "Opfer" oder im Sinne der überholt geglaubten Macht-Hypothese.

Es bleibt als Resümee dieser Abschnitte 2 bis 4 der paradoxe Befund, daß alle durchaus ehrenwerte Aufmerksamkeit, die man dem Rezipienten als Partner, Konsumenten, Abnehmer, jedenfalls als dem unabdingbaren „anderen Ende der Leitung " widmet, wohl eher dazu führt, ihn als Opfer des Medienkonzerts zu erkennen, oder - bei weniger ehrenwerten Motiven, ihn dazu zu machen. Bei diesem Befund muß meines Erachtens der fünfte und letzte Abschnitt einsetzen.

\section{Hat der Leser Pflichten, durch deren Erfüllung er eine Position mindestens der moralischen Stärke aufbauen könnte?}

Wenn er spezifische Pflichten hat, die sich aus seiner Rolle als Rezipient ergeben, dann müssen sie sich aus eben dieser Rolle ableiten lassen. Die pünktliche Bezahlung des Abonnements bzw. der Rundfunkgebühren sind zwar solche spezifischen Pflichten, stehen aber in unserem Zusammenhang nicht infrage. Auch allgemeine Pflichten des Staatsbürgers können meines Erachtens nicht gemeint sein. Denn die Pflicht, sich möglichst vollständig und, wenn's geht, aus verschiedenen Quellen zu informieren, steht zwar dem Recht auf Information gegenüber, - sie ist aber bei unserem Demokratieverständnis eine allgemeine Bürgerpflicht, die nicht erst aus der Tatsache entsteht, daß der Bürger Zeitungsabonnent oder Rundfunk-Teilnehmer ist.

Auch die in der älteren christlichen Medien-Moral stark betonten Christenpflichten „Keine ,schlechten' Zeitungen lesen, am besten gar keine Zeitungen lesen, keine ,schlechten "Filme ansehen" etc. - sind ja in erster Linie moralische Christenpflichten, nicht aber Leser- bzw. Rezipientenpflichten. Glücklicherweise hat spätestens „Communio et progressio" diese Negativ-Ermahnungen durch positive Aufforderungen ersetzt. Das dort, wie schon in "Inter mirifica“ festgestellte Recht auf Information wird u. a. durch die Pflicht, sich zu informieren, inhaltlich näher bestimmt: "Denn das Informationsrecht bliebe nur eine Formel, wenn sich nicht jeder bemüht, auch informiert zu sein." (C e P, Nr. 34).

Die Pastoralinstruktion weist jedoch auch auf einige recht konkrete Pflichten des Rezipienten hin. So soll er z. B. „Verständnis aufbringen für die Situation des Journalisten". Er soll sich möglichst aus mehreren Quellen unterrichten und diese kritisch sichten. (Nr. 41). Er soll sich schließlich über die Medien selbst informieren (Stichwort „Medienpädagogik“, Nr. 64 ff.): „Die Kommunikationsmittel leisten erst dann ihren vollen Beitrag zur Entfaltung des Menschen, wenn er deren Wesen und den Umgang mit ihnen begriffen hat. Wer ihre Bedeutung nur oberflächlich kennt, schmälert leicht den Verfügungsraum seiner Freiheit ${ }^{\star}$ (Nr. 64). 
Paul VI. hat diese Vorstellung in seinem Wort zum letztjährigen Welttag der Kommunikationsmittel in eine umfassende vielleicht etwas zu optimistische Bitte gekleidet: „Die Leser, Hörer und Zuschauer bitten wir, sich einen wachen, kritischen Sinn anzueignen, der sie befähigt, jenen Menschen, Presseerzeugnissen, Sendungen und Filmen mit aufmerksamem Interesse zu begegnen sowie moralische und materielle Unterstïtzung angedeihen zu lassen, welche die Rechte des Menschen verteidigen und der Erziehung zur Erfüllung seiner Pflichten dienlich sind. Zugleich werden sie dann auch in der Lage sein, sich gegen Angriffe und Verführungen zur Wehr zu setzen, die der objektiven Wahrheit und der Würde des Menschen widersprechen. Wir bitten das Angebot in den Medien richtig zu werten und es auch zu lernen, den Medien gegenüber durch geeignete Einzel- und Gruppeninitiativen vorstellig zu werden. Die Leser, Zuschauer und Hörer haben dank ibrer Auswabl stets das letzte Wort über die Zukunft sozialer Kommunikationsmittel, eine Verantwortung, die ihnen oft unbekannt ist. ${ }^{\text {"14 }}$

Diese Verantwortung, punktuell formulierbar in bestimmten „Pflichten“, kann heute, - wer wollte sich darüber hinwegtäuschen? - nur als Lernziel formuliert werden. Als generelles Ziel - gewissermaßen als "Ober-Pflicht" des Lesers - möchte ich die Aufgabe stellen, sich der Rezipientenrolle bewußt zu werden, mindestens zur Kenntnis zu nehmen, daß eine kleine Schicht von Experten, darunter auch Kommunikatoren, schon jetzt viel mehr über die Rezipienten weiß als diese selbst. Ja, diese selbst wissen nicht einmal, daß es verschieden-, aber jedenfalls hochmotivierte Experten gibt, die viel über sie wissen. Es geht also um die Gewinnung eines Rezipienten-Bewußtseins. Freilich nicht um jenes „richtige Bewußtsein “, das so mancher modische Medienpädagoge eilends vorschlug, als, wie es Wolfgang Langenbucher jüngst formuliert hat, „im Zuge der - reichlich späten und dann hektisch-politischen - Wiederentdeckung des Marxismus... auch eine entsprechende Literatur über Medien entstand "15, sondern um ein Wissen vom Sein, vom eigenen Sein als Empfänger, ohne dessen Mitspielen weder die privatwirtschaftlichen noch die öffentlich-rechtlich organisierten Medien sinnvoll funktionieren könnten. Wissen aber auch über das Sein dieser Medien, die Instrumente und Mittler sind, nicht jedoch problemlos anzapfbare Spender allabendlicher Vergnügungen.

Um dieses Wissen zu vermitteln, ist in der Tat „Medienpädagogik “ eine Pflicht. Sich ihr auszusetzen, solange Bonanza oder Peter Alexander so viel Spaß machen, mutet dem Zeitgenossen noch zu viel Askese zu.

Aber ich bin nicht ohne Hoffnung. Irgendwann haben doch unsere Zeitgenossen begreifen gelernt, daß ein Auto keine psycho-physische Potenz-Prothese ist, sondern ein Gerät aus Blech, Draht und Gummi, für bestimmte Fortbewegungszwecke bedingt geeignet; den dafür notwendigen Regeln haben sie sich pflichtmäßig unterworfen. Und irgendwann werden sie auch den Medien des Kaisers neue Kleider auszuziehen beginnen. Die Frage nach dem "Opfer" oder "Partner" ist damit bis heute noch nicht entschieden. Manches, z. B. die an Umfang zunehmenden Leserbriefteile vieler Zeitungen, deuten auf ein wachsendes Rezipienten- und damit Partnerbewußtsein. Zu seiner Pflege und Entwicklung beizutragen, stünde übrigens auch den aktiven Publizisten gut an. Die Versuchung, sich zu Präzeptoren der Nation aufzuschwingen, ist offensichtlich immer noch gegeben, und zwar in einigen Medien sichtbar mehr als in anderen. Aber in einer der Achtung vor den Grundrechten des Herrn Jedermann verpflichteten Gesellschaft sollte man darauf setzen, daß die Rolle des Publizisten sich mehr und mehr zu der des dienstleistenden Mittlers hin wandelt. 
1. CS veröffentlicht diesen Beitrag als erste Vorbereitung auf die Thematik ("Eine Presse für den Menschen ") des XI. Katholischen Weltkongresses der Presse (UCIP; Wien 10.14. 10. 1977). Er beruht auf M. Schmolkes Vortrag "Der Leser - Opfer oder Partner der Medien? Rechte des Lesers, Pflichten des Lesers “ bei der Jahrestagung der Gesellschaft Katholischer Publizisten Deutschlands am 7. Oktober 1976 in München.

2. Hinweise bei H. D. Fischer: Die Zeitung als Forschungsproblem, in H. D. Fischer (Hrsg.): Deutsche Zeitungen des 17. bis 20. Jahrhunderts, Pullach 1972, S. 11-24, hier S. $20 \mathrm{ff}$.

3. [Osterreichisches] Bundesministerium für Justiz (Hrsg.): Regierungsvorlage zum Mediengesetz (54 BlgNR XIV. GP), Wien 1976.

4. J. W. Mole: Freiheit der Information - zwei Jahresgedenktage, in: CS 6:1973, S. 334339.

5. Vgl. die von $H$. Wagner besorgte und kommentierte Ausgabe Pastoralinstruktion "Communio et Progressio" über die Instrumente der sozialen Kommunikation (Nachkonziliare Dokumentation Bd. 11), Trier 1971.

6. „Die sozialen Kommunikationsmittel angesichts der grundlegenden Rechte und Pflichten des Menschen“, hier zit. n. „Schweizerische Kirchenzeitung“, 144. Jg. 1976, Nr. 22, S. 333-336 (vgl. CS 9:1976, 345-351).

7. Vgl. dazu neuerdings die diesem Schwerpunkt gewidmete Nr. 38/1976 der Wochenzeitung „Das Parlament“, gestaltet von Claus Eurich.

8. Feldbefragung. Kommunikations- und Informationserwartungen der katholischen Bevölkerung gegenüber kirchlichen Massenmedien - insbesondere der Kirchenpresse. Wuppertal 1975, 1976 (mehrere nicht-veröffentlichte Bericht- bzw. Kommentarbände): Institut für Kommunikationsforschung e. V.

9. J. W. Naumann (Hrsg.): „Majestät" Leser entscheidet!?, Augsburg 1951.

10. Vgl. D. Stolte (Hrsg.): Das Fernsehen und sein Publikum, Mainz 1973.

11. Vgl. W. Darschin: Kinder vor dem Bildschirm, in: „Media Perspektiven“ 8/76, S. 366370.

12. Vgl. H. Kellner: Fernsehen als Sozialisationsfaktor, in: „Media Perspektiven“ 7/76, S. $297-310$.

13. E. Noelle-Neumann: Die Schweigespirale. Uber die Entstehung der öffentlichen Meinung, in: Forsthoff/Hörstel (Hrsg.): Standorte im Zeitstrom. FS für Arnold Gehlen ..., Frankfurt/M. 1974, S. 299-330, bzw.

dieselbe: Der Einfluß der Massenmedien auf die quasistatistische Wahrnehmung..., in: O. Schatz (Hrsg.): Die elektronische Revolution, Graz 1975, S. 177-196;

dieselbe: Kumulation, Konsonanz und Offentlichkeitseffekt, in: „Publizistik“, 18:1973, S. $26-55$.

14. Vgl. Anm. 6. - Hervorhebung M. S.

15. W. R. Langenbucher: Stellungnahme zur Frage „Medienpädagogik“ - was ist das?, in: "Medien und Erziehung (früher „Jugend, Film, Fernsehen “) 3/76, S. 163 f.

\section{S U M M A R Y}

The role of recipients in Mass Media Communications has been studied up till now mainly by groups having certain interests in them. Voting and consumer habits have been focal points for empirical research on recipients. The World Congress of the Catholic Press in 1977 in Vienna however is supposed to study the role of the press for man. What is the position of man as a recipient? Does he have rights and duties? Is he conscious about his role as recipient? From these questions the author concludes that the recipient is not a powerless victim of Mass Media. He often is put into such a position because he knows very little about his place 
in the communications process and he does not think about it. Schmolke applies this conclusion to five areas. These are the rights of the recipients, economical power of the recipient, research on the recipient, growing interest on the recipient research as well as the responsibilities of the recipient. He feels that with Media Pedagogy one can develop a certain self consciousness of the recipient.

\section{RÉSUME}

La question concernant le "receveur dans le processus de la communication de masse a été posée, jusqu'ici, la plupart du temps par les défenseurs d'intérêts bien définis. La recherche politique en matière électorale et la recherche des consommateurs économiquement motivée ont donné le coup d'envoi décisif à une importante recherche empirique au sujet du »receveur«. En 1977, le congrès mondial de la presse catholique (à Vienne) posera cependant la question d'une "Presse pour l'homme«. Quelle position le receveur a-t-il en tant qu'bomme? A-t-il des droits, a-t-il des devoirs? Aprds tout, a-t-il conscience de son rôle de receveur? L'auteur examine ces questions et émet la thèse suivante: le receveur n'est pas la victime impuissante des mass media, -mais il se laisse souvent et facilment pousser dans ce rôle parce qu'il sait très peu à son sujet (sur son rôle en tant que receveur) et y réfléchit encore moins. Il examine sa thèse dans cinq domaines: Les droits du receveur, le potentiel économique du receveur, l'examen du receveur, l'intérêt croissant pour la recherche sur le receveur et les devoirs du receveur. Il en arrive à la conclusion qu'il faut, au moyen d'une pédagogie des mass media, mettre le receveur au courant et l'amener à une meilleure connaissance de lui-mêne.

\section{R E S U M E N}

La pregunta sobre el »receptor en el proceso de la comunicación social fue planteada hasta ahora a menudo por representantes de muy determinados intereses. Encuestas entre electores y sondeos entre consumidores dieron impulsos esenciales para una ámplia investigación empírica sobre el receptor. El congreso mundial de la prensa católica 1977 (Viena) se preguntará sobre la "prensa para el hombre ". Qué posición ocupa el receptor como hombre? Tiene derechos, deberes? Es consciente siquiera de su papel como receptor? El autor analiza estos interrogantes y formula la tesis: El receptor no es una víctima indefensa ante los medios de difusión, pero a menudo deja que le asignen con facilidad ese papel porque sabe demasiado poco sobre sí mismo (en su papel de receptor) y reflexiona aún menos sobre ello. Analiza su tesis en cinco estadios: Derechos del receptor, potencial económico del receptor, creciente interés por el análisis del receptor, investigación del receptor y deberes del receptor. Llega a la conclusión de que, con ayuda de la pedagogía de los medios de difusión, debe trabajarse en la "mentalización del receptor«. 